\title{
Behaviours and Attitudes to Joint Issues and Osteoarthritis ${ }^{+}$
}

\author{
Cassandra Slade *, Kathryn Beck, Cathryn Conlon and Pamela von Hurst \\ School of Sports, Exercise and Nutrition, Massey University, Auckland 4442, New Zealand; \\ K.L.Beck@massey.ac.nz (K.B.); C.Conlon@massey.ac.nz (C.C.); P.R.vonHurst@massey.ac.nz (P.v.H.) \\ * Correspondence: C.Slade2@massey.ac.nz; Tel.: +64-9-213-6859 \\ † Presented at the 2019 Annual Meeting of the Nutrition Society of New Zealand, Napier, New Zealand, \\ 28-29 November 2019.
}

Published: 20 January 2020

Osteoarthritis (OA) is a major cause of disability worldwide and in New Zealand, and a huge burden on the individual and society. To date effective early diagnosis, prevention and treatment strategies are lacking. This study examined behaviours and attitudes towards joint issues and OA.

Using a composite score from the KOOS (Knee Injury and Osteoarthritis Outcome Score) questionnaire, 183 participants with increased joint issues (scoring in the bottom 2 quartiles) were identified from the REACH study cohort $(n=367)$. These participants were sent a questionnaire on behaviours and attitudes regarding joint issues and OA. Associations between patterns of behavior and attitudes were analysed in the 95 participants (58 females; 37 males) who completed the questionnaire.

Most (87\%) participants experienced joint pain and all experienced one or more joint symptoms even though half $(n=48)$ had no OA diagnosis. Those without a medical diagnosis were significantly more likely to experience little/no pain and little/no impact on lifestyle than those diagnosed $\left(\chi^{2}=\right.$ $8.98, p=0.003 ; \chi^{2}=16.3, p=0.001$ respectively). Increased pain and impact on lifestyle were associated with increased pain relief intake $\left(\chi^{2}=14.3, p=0.001 ; \chi^{2}=15.4, p=0.001\right.$ respectively). Taking supplements and homeopathic remedies were not significantly associated with OA diagnosis, pain or impact on lifestyle. Exercise was the most common therapy (81\% participants) and most had a positive attitude towards all treatments experienced. The majority however hadn't tried a variety of treatments with the attitude that joint issues are "just part of getting old" being the most common barrier (47\%).

Joint symptoms are prevalent even without OA diagnosis and there is reliance on pharmaceutical management of $\mathrm{OA}$ and a lack of knowledge and positive attitudes toward alternative treatment strategies. This highlights the need for the development of early diagnosis techniques, improved management methods and treatments for OA.

(C) 2020 by the authors. Licensee MDPI, Basel, Switzerland. This article is an open access article distributed under the terms and conditions of the Creative Commons Attribution (CC BY) license (http://creativecommons.org/licenses/by/4.0/). 